Supporting Information

\title{
Influence of Organic Ligands on the Reduction of Polyhalogenated Alkanes by Iron(II)
}

\author{
Adam L. Bussan and Timothy J. Strathmann ${ }^{*}$ \\ University of Illinois at Urbana-Champaign \\ Department of Civil and Environmental Engineering \\ 205 North Mathews, Urbana, Illinois, 61801 \\ ${ }^{*}$ Corresponding author e-mail: strthmnn@uiuc.edu
}

July 2007

8 Pages

6 Sections

3 Tables

2 Figures 


\section{Section S1. Reagents}

All chemicals were reagent grade and were used as received from the supplier. $\mathrm{FeCl}_{2} \cdot 4 \mathrm{H}_{2} \mathrm{O}$, $\mathrm{NaCl}, \mathrm{NaOH}$, MES buffer (4-morpholineethanesulfonic acid; $\left.\mathrm{p} K_{\mathrm{a}}=6.10\right)$, MOPS buffer (3-(Nmorpholino)propanesulfonic acid; $\left.\mathrm{p} K_{\mathrm{a}}=7.20\right)$, EPPS buffer (4-(2-hydroxyethyl)-1piperazinepropanesulfonic acid; $\left.\mathrm{p} K_{\mathrm{a}}=8.0\right)$, citric acid monohydrate, EDTA (ethylenediaminetetraacetic acid, disodium salt), L-cysteine ((R)-2-amino-3-mercaptopropionic acid), 2,3-DMSA (meso-2,3-dimercaptosuccinic acid), pyromellitic acid (1,2,4,5benzenetetracarboxylic acid), sodium salicylate, 2,3-DHBA (2,3-dihydroxybenzoic acid), 3,4DHBA (3,4-dihydroxybenzoic acid), 2,3,4-THBA (2,3,4-trihydroxybenzoic acid), 3,4,5-THBA (3,4,5-trihydroxybenzoic acid), thioglycolic acid, 1,1,1-trichloroethane $\left(\mathrm{CH}_{3}-\mathrm{CCl}_{3}\right), 1,1,2,2$ tetrachloroethane $\left(\mathrm{CHCl}_{2}-\mathrm{CHCl}_{2}\right)$, 1,1,1,2-tetrachloroethane $\left(\mathrm{CH}_{2} \mathrm{Cl}_{-}-\mathrm{CCl}_{3}\right)$, pentachloroethane $\left(\mathrm{CHCl}_{2}-\mathrm{CCl}_{3}\right)$, 1-chloro-3-fluorobenzene, chlorodibromomethane $\left(\mathrm{CHBr}_{2} \mathrm{Cl}\right)$, bromotrichloromethane $\left(\mathrm{CBrCl}_{3}\right)$, hexachloroethane $\left(\mathrm{CCl}_{3}-\mathrm{CCl}_{3}\right)$, carbon tetrachloride $\left(\mathrm{CCl}_{4}\right)$, bromodichloromethane $\left(\mathrm{CHBrCl}_{2}\right)$, tribromomethane $\left(\mathrm{CHBr}_{3}\right)$, sulfuric acid, potassium hydrogen phthalate, 2',4',5'-trifluoroacetophenone, methanol, acetonitrile, acetaldehyde, and o-(2,3,4,5,6pentafluorobenzyl)hydroxylamine hydrochloride were obtained from Sigma-Aldrich-FlukaSupelco. $\mathrm{HCl}$, hexane, and TAPS buffer ( $N$-[tris(hydroxymethyl)methyl]-3aminopropanesulfonic acid; $\left.\mathrm{p} K_{\mathrm{a}}=8.40\right)$ were purchased from Fisher. Tiron $(1,2-$ dihydroxybenzene-3,5-disulfonic acid, disodium salt) was obtained from Avocado Organics. nHeptane was obtained from Acros Organics. 1,1,1-Trichlorotrifluoroethane $\left(\mathrm{CF}_{3}-\mathrm{CCl}_{3}\right)$, tetrachloro-1,1-difluoroethane $\left(\mathrm{CF}_{2} \mathrm{Cl}-\mathrm{CCl}_{3}\right)$, and fluorotribromomethane $\left(\mathrm{CFBr}_{3}\right)$ were obtained from Synquest Labs. Gas standards for ethene, ethane, and $n$-butane were supplied by Scott Specialty Gases.

\section{Section S2. Gas Chromatography Methods}

Headspace samples from 1,1,1-TCA reactions were analyzed with a Hewlett-Packard 6890 gas chromatograph with flame ionization detector (GC-FID; detector temp $250^{\circ} \mathrm{C}$ ) and a GS-Q column $(0.53 \mathrm{~mm}$ i.d. $\times 30 \mathrm{~m}$; J\&W Scientific). Samples were injected in splitless mode with the injector at $250^{\circ} \mathrm{C}$, and $\mathrm{He}$ was used as the carrier gas. The oven temperature was programmed at $50^{\circ} \mathrm{C}$ for the first $2 \mathrm{~min}$, then ramped to $200^{\circ} \mathrm{C}$ at $25^{\circ} \mathrm{C} / \mathrm{min}$ and held constant for an additional 2 minutes. Reactions with other PHAs were analyzed with a Hewlett-Packard 5890 $\mathrm{GC}$ with electron capture detector (GC-ECD; $250^{\circ} \mathrm{C}$ detector temp) and a DB-624 column ( 0.53 $\mathrm{mm}$ i.d. $\times 30 \mathrm{~m}$; J\&W Scientific). Samples were injected in splitless mode with the injector at $220^{\circ} \mathrm{C}$, and $\mathrm{He}$ was used as the carrier gas. The oven temperature was programmed at $50^{\circ} \mathrm{C}$ for the first minute, then increased to $85^{\circ} \mathrm{C}$ at $5^{\circ} \mathrm{C} / \mathrm{min}$ to. The GC-FID and GC-ECD systems were calibrated by equilibrating known concentrations of PHAs and internal standard ( $n$-heptane for GC-FID; 1-chloro-3-fluorobenzene GC-ECD) in sealed serum vials containing the same ratio of aqueous and gas phase as the samples being analyzed, thereby accounting for aqueous-gas partitioning and enabling determination of concentrations in terms of moles per vial.

Acetaldehyde was analyzed with a Varian 4000 gas chromatograph-mass spectrometer (GCMS) using a method adapted from EPA Method 556. Quenched reaction aliquots $(4 \mathrm{~mL})$ were derivatized with pentafluorobenzylhydroxylamine $(0.8 \mathrm{~mL} 15 \mathrm{mg} / \mathrm{l})$ for $2 \mathrm{hrs}$ at $37^{\circ} \mathrm{C}$, then extracted with $4 \mathrm{~mL}$ of hexane, of which $5 \mu \mathrm{l}$ was analyzed by GC-MS (injector temp $=200{ }^{\circ} \mathrm{C}$ ). The column $\left(0.25 \mathrm{~mm}\right.$ i.d. $\times 30 \mathrm{~m}$, Varian Factor Four) was held at $60^{\circ} \mathrm{C}$ for the first $2 \mathrm{~min}$, then increased to $130^{\circ} \mathrm{C}$ at $7^{\circ} \mathrm{C} / \mathrm{min}$. The mass spectrometer was operated under full scan electron impact mode. 
Section S3. Results of Batch Kinetic Experiments

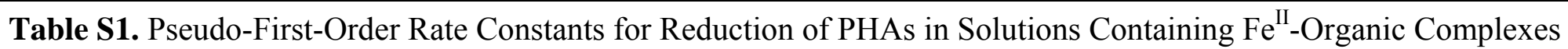

\begin{tabular}{|c|c|c|c|c|c|c|c|c|}
\hline PHA Identity & Ligand Identity & Setup & $\mathrm{pH}$ & $\begin{array}{c}{[\mathrm{PHA}]_{\mathrm{o}}} \\
(\mu \mathrm{M})\end{array}$ & $\begin{array}{l}{\left[\mathrm{Fe}^{\mathrm{II}}\right]} \\
(\mathrm{mM})\end{array}$ & $\begin{array}{c}\text { [Ligand] } \\
(\mathrm{mM})\end{array}$ & $\begin{array}{c}\mathrm{I}^{a} \\
(\mathrm{M}) \\
\end{array}$ & $\begin{array}{c}k_{\text {obs }}^{\prime} b \\
\left(\mathrm{~s}^{-1}\right)\end{array}$ \\
\hline \multirow{26}{*}{$\begin{array}{c}\mathrm{CH}_{3}-\mathrm{CCl}_{3} \\
(1,1,1-\mathrm{TCA})\end{array}$} & \multirow{16}{*}{ Tiron } & HS & 7.02 & 50 & 0.5 & 10 & 0.25 & $1.38( \pm 0.18) \times 10^{-6}$ \\
\hline & & HS & 7.30 & 50 & 0.5 & 10 & 0.25 & $7.55( \pm 0.59) \times 10^{-6}$ \\
\hline & & HS & 7.56 & 50 & 0.5 & 10 & 0.25 & $1.49( \pm 0.12) \times 10^{-5}$ \\
\hline & & HS & 7.77 & 50 & 0.5 & 10 & 0.25 & $2.23( \pm 0.33) \times 10^{-5}$ \\
\hline & & HS & 7.94 & 50 & 0.5 & 10 & 0.25 & $2.87( \pm 0.23) \times 10^{-5}$ \\
\hline & & HS & 8.24 & 50 & 0.5 & 10 & 0.25 & $4.60( \pm 0.27) \times 10^{-5}$ \\
\hline & & HS & 8.52 & 50 & 0.5 & 10 & 0.25 & $5.71( \pm 0.46) \times 10^{-5}$ \\
\hline & & HS & 8.76 & 50 & 0.5 & 10 & 0.25 & $6.45( \pm 0.24) \times 10^{-5}$ \\
\hline & & HS & 8.94 & 50 & 0.5 & 10 & 0.25 & $7.43( \pm 0.43) \times 10^{-5}$ \\
\hline & & ZHS & 9.00 & 50 & 0.5 & 10 & 0.25 & $1.28( \pm 0.17) \times 10^{-4}$ \\
\hline & & HS & 7.48 & 50 & 0.5 & 0.5 & 0.25 & $1.76( \pm 1.64) \times 10^{-7}$ \\
\hline & & HS & 7.51 & 50 & 0.5 & 1 & 0.25 & $7.45( \pm 1.28) \times 10^{-7}$ \\
\hline & & HS & 7.51 & 50 & 0.5 & 5 & 0.25 & $8.97( \pm 0.64) \times 10^{-6}$ \\
\hline & & HS & 7.56 & 50 & 0.5 & 10 & 0.25 & $1.49( \pm 0.12) \times 10^{-5}$ \\
\hline & & HS & 7.49 & 50 & 0.5 & 20 & 0.25 & $1.63( \pm 0.34) \times 10^{-5}$ \\
\hline & & HS & 7.49 & 50 & 0.5 & 50 & 0.25 & $1.69( \pm 0.25) \times 10^{-5}$ \\
\hline & \multirow[t]{10}{*}{ 3,4-DHBA } & HS & 7.42 & 50 & 0.5 & 10 & 0.25 & $4.73( \pm 0.42) \times 10^{-7}$ \\
\hline & & HS & 7.93 & 50 & 0.5 & 10 & 0.25 & $9.48( \pm 0.55) \times 10^{-6}$ \\
\hline & & HS & 8.46 & 50 & 0.5 & 10 & 0.25 & $8.78( \pm 0.46) \times 10^{-5}$ \\
\hline & & HS & 8.87 & 50 & 0.5 & 10 & 0.25 & $2.45( \pm 0.08) \times 10^{-4}$ \\
\hline & & HS & 7.93 & 50 & 0.5 & 0.5 & 0.25 & $1.22( \pm 0.59) \times 10^{-7}$ \\
\hline & & HS & 7.93 & 50 & 0.5 & 1 & 0.25 & $3.60( \pm 1.18) \times 10^{-7}$ \\
\hline & & HS & 7.94 & 50 & 0.5 & 5 & 0.25 & $3.91( \pm 0.57) \times 10^{-6}$ \\
\hline & & HS & 7.93 & 50 & 0.5 & 10 & 0.25 & $9.48( \pm 0.55) \times 10^{-6}$ \\
\hline & & HS & 7.93 & 50 & 0.5 & 20 & 0.25 & $1.89( \pm 0.09) \times 10^{-5}$ \\
\hline & & $\mathrm{HS}$ & 7.93 & 50 & 0.5 & 50 & 0.25 & $2.95( \pm 0.17) \times 10^{-5}$ \\
\hline
\end{tabular}




\begin{tabular}{|c|c|c|c|c|c|c|c|c|}
\hline PHA Identity & Ligand Identity & Setup & $\mathrm{pH}$ & $\begin{array}{c}{[\mathrm{PHA}]_{\mathrm{o}}} \\
(\mu \mathrm{M})\end{array}$ & $\begin{array}{l}{\left[\mathrm{Fe}^{\mathrm{II}}\right]} \\
(\mathrm{mM})\end{array}$ & $\begin{array}{c}\text { [Ligand] } \\
(\mathrm{mM})\end{array}$ & $\begin{array}{c}\mathrm{I}^{a} \\
(\mathrm{M}) \\
\end{array}$ & $\begin{array}{l}k_{\text {obs }}^{\prime} \\
\left(\mathrm{s}^{-1}\right)\end{array}$ \\
\hline \multirow{22}{*}{$\begin{array}{c}\mathrm{CH}_{3}-\mathrm{CCl}_{3} \\
(1,1,1-\mathrm{TCA})\end{array}$} & \multirow[t]{10}{*}{ 3,4,5-ТHBA } & HS & 7.49 & 50 & 0.5 & 10 & 0.25 & $3.70( \pm 0.49) \times 10^{-7}$ \\
\hline & & HS & 7.95 & 50 & 0.5 & 10 & 0.25 & $2.58( \pm 0.31) \times 10^{-6}$ \\
\hline & & HS & 8.56 & 50 & 0.5 & 10 & 0.25 & $7.66( \pm 4.25) \times 10^{-5}$ \\
\hline & & HS & 9.25 & 50 & 0.5 & 10 & 0.25 & $2.06( \pm 0.23) \times 10^{-4}$ \\
\hline & & HS & 7.96 & 50 & 0.5 & 0.5 & 0.25 & $1.63( \pm 1.31) \times 10^{-7}$ \\
\hline & & HS & 7.95 & 50 & 0.5 & 1 & 0.25 & $2.47( \pm 2.27) \times 10^{-7}$ \\
\hline & & HS & 7.95 & 50 & 0.5 & 5 & 0.25 & $2.03( \pm 0.38) \times 10^{-6}$ \\
\hline & & HS & 7.95 & 50 & 0.5 & 10 & 0.25 & $2.58( \pm 0.31) \times 10^{-6}$ \\
\hline & & HS & 7.96 & 50 & 0.5 & 20 & 0.25 & $1.60( \pm 0.40) \times 10^{-5}$ \\
\hline & & HS & 7.95 & 50 & 0.5 & 50 & 0.25 & $3.08( \pm 0.67) \times 10^{-5}$ \\
\hline & \multirow[t]{12}{*}{ 2,3-DMSA } & HS & 6.57 & 50 & 0.5 & 10 & 0.25 & $5.32( \pm 8.83) \times 10^{-6}$ \\
\hline & & HS & 7.01 & 50 & 0.5 & 10 & 0.25 & $9.80( \pm 7.11) \times 10^{-6}$ \\
\hline & & HS & 7.46 & 50 & 0.5 & 10 & 0.25 & $3.23( \pm 1.77) \times 10^{-5}$ \\
\hline & & HS & 8.00 & 50 & 0.5 & 10 & 0.25 & $7.74( \pm 1.93) \times 10^{-5}$ \\
\hline & & HS & 8.56 & 50 & 0.5 & 10 & 0.25 & $1.51( \pm 0.14) \times 10^{-4}$ \\
\hline & & HS & 9.10 & 50 & 0.5 & 10 & 0.25 & $1.38( \pm 0.13) \times 10^{-4}$ \\
\hline & & HS & 8.00 & 50 & 0.5 & 0.5 & 0.25 & $9.33( \pm 0.61) \times 10^{-5}$ \\
\hline & & HS & 7.99 & 50 & 0.5 & 1 & 0.25 & $1.60( \pm 0.17) \times 10^{-4}$ \\
\hline & & HS & 7.99 & 50 & 0.5 & 5 & 0.25 & $1.01( \pm 0.06) \times 10^{-4}$ \\
\hline & & HS & 8.00 & 50 & 0.5 & 10 & 0.25 & $7.74( \pm 1.93) \times 10^{-5}$ \\
\hline & & HS & 7.99 & 50 & 0.5 & 20 & 0.25 & $2.15( \pm 0.38) \times 10^{-5}$ \\
\hline & & HS & 7.99 & 50 & 0.5 & 40 & 0.25 & $1.07( \pm 0.74) \times 10^{-5}$ \\
\hline
\end{tabular}


Table S1. Continued

\begin{tabular}{|c|c|c|c|c|c|c|c|c|}
\hline PHA Identity & Ligand Identity & Setup & $\mathrm{pH}$ & $\begin{array}{c}{[\mathrm{PHA}]_{\mathrm{o}}} \\
(\mu \mathrm{M})\end{array}$ & $\begin{array}{l}{\left[\mathrm{Fe}^{\mathrm{II}}\right]} \\
(\mathrm{mM})\end{array}$ & $\begin{array}{c}\text { [Ligand] } \\
(\mathrm{mM})\end{array}$ & $\begin{array}{c}\mathrm{I}^{a} \\
(\mathrm{M})\end{array}$ & $\begin{array}{c}k_{\text {obs }}^{\prime} b \\
\left(\mathrm{~s}^{-1}\right)\end{array}$ \\
\hline $\mathrm{CH}_{3}-\mathrm{CCl}_{3}$ & Tiron & ZHS & 7.55 & 50 & 0.5 & 10 & 0.25 & $2.98( \pm 0.21) \times 10^{-5}$ \\
\hline $\mathrm{CH}_{2} \mathrm{Cl}-\mathrm{CCl}_{3}$ & & ZHS & 7.56 & 1 & 0.5 & 10 & 0.25 & $3.73( \pm 0.36) \times 10^{-4}$ \\
\hline $\mathrm{CHCl}_{2}-\mathrm{CCl}_{3}$ & & ZHS & 7.56 & 1 & 0.5 & 10 & 0.25 & $1.46( \pm 0.18) \times 10^{-3}$ \\
\hline $\mathrm{CCl}_{3}-\mathrm{CCl}_{3}$ & & ZHS & 7.58 & 0.1 & 0.5 & 10 & 0.25 & $5.94( \pm 0.86) \times 10^{-3}$ \\
\hline $\mathrm{CHCl}_{2}-\mathrm{CHCl}_{2}$ & & ZHS & 7.59 & 50 & 0.5 & 10 & 0.25 & $3.41( \pm 0.91) \times 10^{-6}$ \\
\hline $\mathrm{CF}_{3}-\mathrm{CCl}_{3}$ & & ZHS & 7.56 & 0.1 & 0.5 & 10 & 0.25 & $8.21( \pm 1.27) \times 10^{-4}$ \\
\hline $\mathrm{CF}_{2} \mathrm{Cl}-\mathrm{CCl}_{3}$ & & ZHS & 7.56 & 0.1 & 0.5 & 10 & 0.25 & $1.81( \pm 0.23) \times 10^{-3}$ \\
\hline $\mathrm{CCl}_{4}$ & & ZHS & 7.56 & 0.1 & 0.5 & 10 & 0.25 & $6.36( \pm 0.27) \times 10^{-3}$ \\
\hline $\mathrm{CHBrCl}_{2}$ & & ZHS & 7.56 & 1 & 0.5 & 10 & 0.25 & $2.45( \pm 0.17) \times 10^{-3}$ \\
\hline $\mathrm{CHBr}_{2} \mathrm{Cl}$ & & ZHS & 7.54 & 1 & 0.5 & 10 & 0.25 & $4.22( \pm 0.63) \times 10^{-3}$ \\
\hline $\mathrm{CHBr}_{3}$ & & ZHS & 7.58 & 10 & 0.5 & 10 & 0.25 & $4.58( \pm 0.26) \times 10^{-3}$ \\
\hline $\mathrm{CBr}_{2} \mathrm{Cl}_{2}$ & & ZHS & 6.51 & 50 & 0.5 & 10 & 0.25 & $8.22( \pm 0.86) \times 10^{-2}$ \\
\hline $\mathrm{CBrCl}_{3}$ & & ZHS & 6.49 & 20 & 0.5 & 10 & 0.25 & $2.97( \pm 0.39) \times 10^{-2}$ \\
\hline $\mathrm{CFBr}_{3}$ & & ZHS & 6.52 & 50 & 0.5 & 10 & 0.25 & $4.34( \pm 0.41) \times 10^{-3}$ \\
\hline
\end{tabular}

${ }^{a}$ Ionic strength set by combination of ionic solution constituents (buffer, ligand, $\mathrm{NaCl}$ ). ${ }^{b}$ Uncertainty represents $95 \%$ confidence interval, determined from linear regression data fit with a pseudo first-order rate expression (eq 3). HS = headspace reactor setup. ZHS = zero headspace reactor setup. 


\section{Section S4. Survey of Reactivity of Representative Fe"-Ligand Complexes}

A survey experiment was initially conducted to identify natural ligand donor groups that form $\mathrm{Fe}^{\mathrm{II}}$ complexes capable of reducing PHAs. 1,1,1-TCA was spiked into a series of batch reactors containing $\mathrm{Fe}^{\mathrm{II}}(0.5 \mathrm{mM})$ and individual low molecular weight organic ligands $(10 \mathrm{mM})$ possessing different Lewis base donor functional groups contained in natural organic matter (e.g., carboxylic, amino, thiol, phenolic). In comparison to a buffer-only blank reaction, no significant loss of 1,1,1-TCA is observed over $1000 \mathrm{~h}$ in reactors containing only $\mathrm{Fe}^{\mathrm{II}}$ (ligandfree control) or individual ligands ( $\mathrm{Fe}^{\mathrm{II}}$-free controls). Figure S1 shows the concentration of 1,1,1-TCA remaining after 10,100, and 1000 hours in solutions containing $\mathrm{Fe}^{\mathrm{II}}$ and several of the ligands that were surveyed. Ligands that combine with $\mathrm{Fe}^{\mathrm{II}}$ to promote declines in 1,1,1TCA concentration that are significantly greater than observed buffer-only control are shown in Figure 1B. Comparison of the ligand structures reveals that reactive $\mathrm{Fe}^{\mathrm{II}}$ complexes are formed with those containing either catechol or organothiol donor groups in their structures, whereas $\mathrm{Fe}^{\text {II }}$ complexes with ligands lacking these groups (e.g., citric acid, EDTA, salicylic acid) do not reduce 1,1,1-TCA. This finding agrees with earlier work in our lab examining the reactivity of $\mathrm{Fe}^{\mathrm{II}}$-organic complexes with nitroaromatic and $N$-heterocyclic nitramine contaminants $(1,2)$.

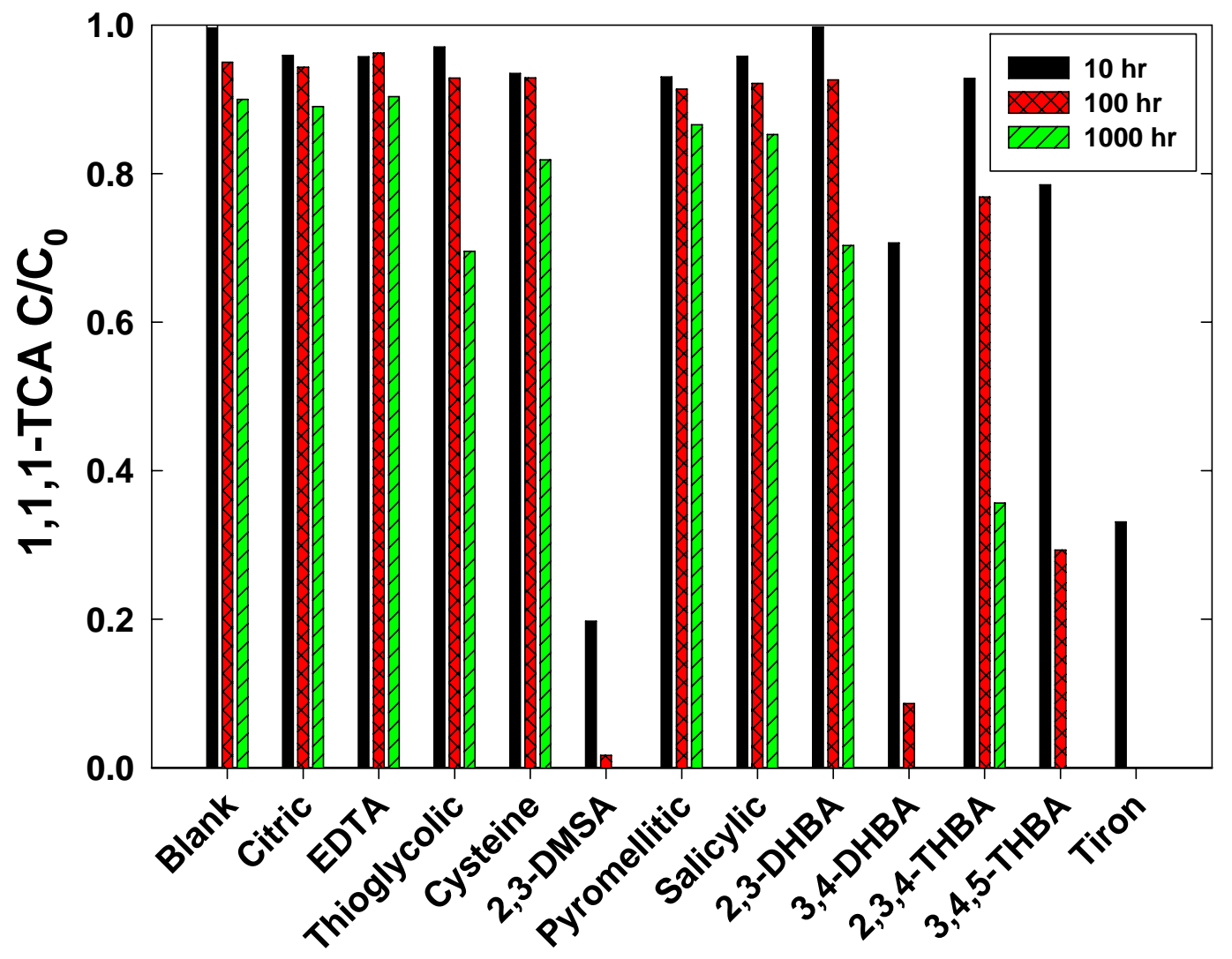

Figure S1. Influence of different organic ligands on Fe" reactivity with 1,1,1-TCA. Chart shows the concentration of 1,1,1-TCA remaining after reacting for 10,100, and 1000 hours in solutions containing Fe" plus representative organic ligands. Initial reaction conditions: $50 \mu \mathrm{M}$ 1,1,1-TCA, $0.5 \mathrm{mM} \mathrm{Fe}$ ", $10 \mathrm{mM}$ ligand, pH 8.0 (50 mM EPPS buffer). Ligand abbreviations: EDTA = ethylenediaminetetraacetic acid; 2,3-DMSA = meso-2,3-dimercaptosuccinic acid; DHBA = dihydroxybenzoic acid; THBA = trihydroxybenzoic acid. 1,1,1-TCA disappearance in Fe"-free solutions (ligand-only controls) and ligand-free solutions (Fe"-only control) is not significantly different from the blank (buffer-only control). 


\section{Section S5. Fe" Speciation in Tiron Solutions}
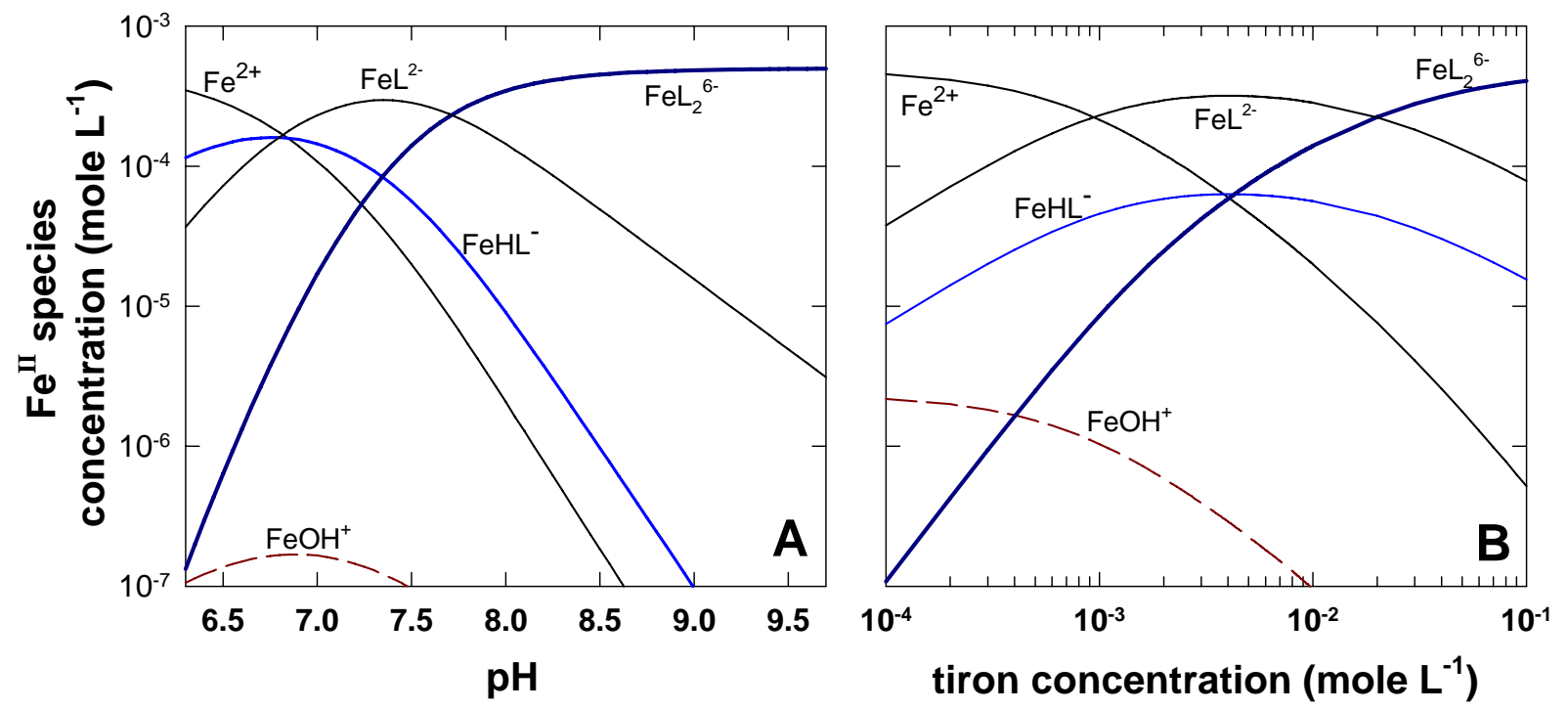

Figure S2. Calculated speciation of $0.5 \mathrm{mM} \mathrm{Fe}$ il in aqueous solution containing tiron at conditions corresponding to the kinetic studies shown in Figure 2. Panel A shows the effects of varying $\mathrm{pH}$ in solution containing $10 \mathrm{mM}$ tiron, and panel $\mathrm{B}$ shows the effects of varying tiron concentration at $\mathrm{pH} 7.5$. Calculations performed using the speciation model provided in Table S2, assuming $\mathrm{I}=0.25 \mathrm{M}$ and $\mathrm{T}=25^{\circ} \mathrm{C}$.

Table S2. Equilibrium Speciation Model for Fe $\mathrm{FI}^{\mathrm{II}}$ Tiron Solutions ${ }^{a}$

\begin{tabular}{cc}
\hline Hydrolysis & $\log \boldsymbol{K}(\mathbf{I}=\mathbf{0})$ \\
$\mathrm{H}_{2} \mathrm{O}-\mathrm{H}^{+}=\mathrm{OH}^{-}$ & $-14.00^{b}$ \\
$\mathrm{Fe}^{2+}+\mathrm{H}_{2} \mathrm{O}-\mathrm{H}^{+}=\mathrm{FeOH}$ & $-9.40^{b}$ \\
$\mathrm{Fe}^{2+}+2 \mathrm{H}_{2} \mathrm{O}-2 \mathrm{H}^{+}=\mathrm{Fe}(\mathrm{OH})_{2}{ }^{-}$ & $-20.49^{b}$ \\
$\mathrm{Fe}^{2+}+3 \mathrm{H}_{2} \mathrm{O}-3 \mathrm{H}^{+}=\mathrm{Fe}(\mathrm{OH})_{3}{ }^{-}$ & $-28.99^{b}$ \\
Acid-Base Reactions & \\
$\mathrm{L}^{4-}+\mathrm{H}^{+}=\mathrm{HL}^{3-}$ & $13.36^{b}$ \\
$\mathrm{~L}^{4-}+2 \mathrm{H}^{+}=\mathrm{H}_{2} \mathrm{~L}^{2-}$ & $21.62^{b}$ \\
$\mathbf{F e}^{\mathbf{I I}} \mathbf{C o m p l e x a t i o n}^{2+}$ & \\
$\mathrm{Fe}^{2+}+\mathrm{L}^{4-}=\mathrm{FeL}^{2-}$ & $10.63^{c}$ \\
$\mathrm{Fe}^{2+}+\mathrm{H}^{+}+\mathrm{L}^{4-}=\mathrm{FeHL}^{-}$ & $17.85^{c}$ \\
$\mathrm{Fe}^{2+}+2 \mathrm{~L}^{4-}=\mathrm{FeL}_{2}^{6-}$ & $15.33^{c}$ \\
\hline
\end{tabular}

${ }^{a} \log K$ values corrected to $\mathrm{I}=0$ using the Davies Equation. ${ }^{b}$ From ref (3). ${ }^{c}$ From ref (1). 


\section{Section S6. Linear Free Energy Relationships}

Table S3. Calculated Bimolecular Rate Constants for Reduction of PHAs by the 1:2 Fe(II)-Tiron Complex and Molecular Descriptors used for LFER Analysis. ${ }^{a}$

\begin{tabular}{ccccc}
\hline $\mathrm{PHA}$ & $\begin{array}{c}k_{\mathrm{FeL}}^{6-}{ }^{\mathrm{b}} \\
\left(\mathrm{M}^{-1} \mathrm{~s}^{-1}\right)\end{array}$ & $\begin{array}{c}\Delta G^{0^{\prime} \mathrm{c}} \\
\left(\mathrm{kJ} \mathrm{mol}^{-1}\right)\end{array}$ & $\begin{array}{c}D_{\mathrm{R}-\mathrm{X}}{ }^{\mathrm{d}} \\
\left(\mathrm{kJ} \mathrm{mol}^{-1}\right)\end{array}$ & $\begin{array}{c}\mathrm{E}_{\mathrm{LUMO}}{ }^{\mathrm{e}} \\
\left(\mathrm{kJ} \mathrm{mol}^{-1}\right)\end{array}$ \\
\hline $\mathrm{CH}_{3}-\mathrm{CCl}_{3}$ & $9.48( \pm 1.28) \times 10^{-2}$ & 47.8 & 288.2 & -243.3 \\
$\mathrm{CH}_{2} \mathrm{Cl}_{-}-\mathrm{CCl}_{3}$ & $2.25( \pm 0.22) \times 10^{0}$ & 32.0 & 270.5 & -316.5 \\
$\mathrm{CHCl}_{2}-\mathrm{CCl}_{3}$ & $8.80( \pm 1.08) \times 10^{0}$ & 22.2 & 262.2 & -332.7 \\
$\mathrm{CCl}_{3}-\mathrm{CCl}_{3}$ & $3.42( \pm 0.49) \times 10^{1}$ & 10.2 & 259.4 & -340.6 \\
$\mathrm{CHCl}_{2}-\mathrm{CHCl}_{2}$ & $1.90( \pm 0.51) \times 10^{-2}$ & 54.0 & 294.2 & -280.3 \\
$\mathrm{CF}_{3}-\mathrm{CCl}_{3}$ & $4.95( \pm 0.76) \times 10^{0}$ & 28.1 & 272.2 & -359.4 \\
$\mathrm{CF}_{2} \mathrm{Cl}_{-}-\mathrm{CCl}_{3}$ & $1.09( \pm 0.14) \times 10^{1}$ & 20.0 & 264.5 & -350.7 \\
$\mathrm{CCl}_{4}$ & $3.83( \pm 0.16) \times 10^{1}$ & 19.6 & 263.4 & -437.1 \\
$\mathrm{CHBrCl}_{2}$ & $1.48( \pm 0.10) \times 10^{1}$ & 70.5 & 262.1 & -393 \\
$\mathrm{CHBr}_{2} \mathrm{Cl}$ & $2.69( \pm 0.40) \times 10^{1}$ & 69.7 & 261.3 & -443.8 \\
$\mathrm{CHBr}_{3}$ & $2.63( \pm 0.15) \times 10^{1}$ & 66.2 & 260.5 & -483.6 \\
$\mathrm{CBr}_{2} \mathrm{Cl}_{2}$ & $1.19( \pm 0.13) \times 10^{5}$ & 45.6 & 238.2 & -558.7 \\
$\mathrm{CBrCl}_{3}$ & $4.99( \pm 0.65) \times 10^{4}$ & 54.3 & 240.1 & -513.1 \\
$\mathrm{CFBr}_{3}$ & $5.83( \pm 0.56) \times 10^{3}$ & 53.2 & 253.5 & -561.4 \\
\hline
\end{tabular}

${ }^{a}$ Computationally derived molecular descriptors from Perlinger and coworkers $(4,5){ }^{b}$ Bimolecular rate constants for PHA reaction with the 1:2 $\mathrm{Fe}^{\mathrm{II}}$-tiron complex. ${ }^{\circ}$ Free energy of one-electron transfer, including correction for changes in electrostatic effects. ${ }^{\mathrm{d}}$ Corrected bond dissociation energy for weakest C-halogen bond. ${ }^{\mathrm{e}}$ Energy of the lowest unoccupied molecular orbital.

\section{References Cited in Supporting Information}

(1) Naka, D.; Kim, D.; Strathmann, T. J. Abiotic Reduction of Nitroaromatic Compounds by Aqueous Iron(II)-Catechol Complexes. Environ. Sci. Technol. 2006, 40, 3006-3012.

(2) Kim, D.; Strathmann, T. J. Role of Organically Complexed Iron(II) Species in the Reductive Transformation of RDX in Anoxic Environments. Environ. Sci. Technol. 2007, 41, 1257-1264.

(3) Martell, A.E.; Smith, R.M.; Motekaitis, R.J. Critically Selected Stability Constants of Metal Complexes Database, Version 4.0; U.S. Department of Commerce, National Institute of Standards and Technology: Gaithersbug, MD, 1997.

(4) Perlinger, J. A.; Venkatapathy, R.; Harrison, J. F. Linear Free Energy Relationships for Polyhalogenated Alkane Transformation by Electron-Transfer Mediators in Model Aqueous Systems. J. Phys. Chem. A 2000, 2000, 2752-2763.

(5) Perlinger, J. A.; Buschmann, J.; Angst, W.; Schwarzenbach, R. P. Iron Porphyrin and Mercaptojuglone Mediated Reduction of Polyhalogenated Methanes and Ethanes in Homogeneous Aqueous Solution. Environ. Sci. Technol. 1998, 32, 2431-2437. 